\title{
Qualidade do carvão vegetal para o consumo doméstico
}

\section{Quality of charcoal for domestic consumption}

\author{
Rafael Amorim Rosa ${ }^{*}$, Marina Donária Chaves Arantes ${ }^{1}$, Juarez Benigno Paes ${ }^{1}$, Wendel \\ Sandro de Paula Andrade ${ }^{1}$ e Jordão Cabral Moulin ${ }^{1}$
}

${ }^{1}$ Departamento de Engenharia Florestal; Centro de Ciência Agrárias da Universidade Federal do Espírito Santo; 29550-000; Jerônimo Monteiro - ES - Brasil.

\begin{abstract}
The present study aimed to analyze the quality of charcoal, for home consumption, marketed in the municipalities of Espírito Santo State: Alegre, Jerônimo Monteiro e Cachoeiro de Itapemirim. Were analyzed some characteristics of charcoal, such as apparent relative density, moisture, volatile materials content, ash content, fixed carbon content, calorific value, elemental analysis and the results were compared with the parameters required by the Seal Premium of Sao Paulo State. It was found that only one brand in the municipality of Alegre and another brand in the municipality of Jerônimo Monteiro has not submitted satisfactory quality. In the municipality of Cachoeiro de Itapemirim, all samples showed better physical and chemical qualities, on average, compared to charcoal sold in the other two, however, did not meet specifications Seal Premium due to high humidity showed.
\end{abstract}

Key-words: Charcoal, quality and consumption

\section{INTRODUÇÃO}

Para qualificar e predizer uma melhor utilização do carvão vegetal é necessário que este seja avaliado. Para isso, existem procedimentos e normas a serem seguidos; como exemplos têm-se a análise química imediata, elementar e poder calorífico.

O carvão vegetal para ser considerado de boa qualidade para o uso doméstico, deve reunir algumas características como: alta densidade relativa aparente; alto teor de carbono fixo; alto poder calorífico; baixa umidade; baixo teor de materiais voláteis e baixo teor de cinzas (Ribeiro e Vale, 2006).

Todavia, a qualidade do carvão vegetal para o uso doméstico é duvidosa, pois é fato que o controle da carbonização é difícil na maioria dos fornos, produzindo um material heterogêneo, diferindo principalmente em densidade, umidade, composição química, friabilidade, resistência mecânica, reatividade e higroscopicidade (Coutinho e Ferraz, 1988). Pennise et al. (2001) afirmaram que o processo de carbonização libera em sua fumaça mais de 130 substâncias tóxicas, sendo 10 delas classificadas como hidrocarbonetos policíclicos aromáticos (HPA's), que podem causar câncer, mutações genéticas e desregular o sistema endócrino.

Visando determinar o padrão de qualidade do carvão vegetal, tais como teor de carbono fixo, teor de materiais voláteis e teor de cinzas, práticas de processamento, faixa de tolerância para o tamanho das peças de carvão vegetal embalado, tipo preferencial de embalagem, a origem do produto e intervalos de tolerância, foi criado em 2003 no Estado de São Paulo o Selo Premium, promulgado pela a Resolução $n^{\circ} 10 \mathrm{SAA}$, de 11 de julho de 2003. Ainda nesta resolução, a umidade do carvão vegetal deve estar abaixo de 5\%, o teor de carbono fixo (TCF) deve ser maior que $75 \%$, teor de materiais voláteis (TMV) e o teor de cinzas devem ser menores que $23,5 \%$ e $1,5 \%$, respectivamente.

No que diz respeito ao uso da lenha no Estado do Espírito Santo, o Instituto Capixaba de Pesquisa, Assistência Técnica e Extensão Rural/ INCAPER [2003?], divulgou que 24.000 residências capixabas dependem dessa fonte de energia para o

Author for correspondence: rafaelrosa.001@gmail.com

J. Biotec. Biodivers. v. 3, N.2: pp. 41-48, May. 2012 
preparo de alimentos, o que representa $20 \%$ das casas rurais do Estado, além disso, havia a necessidade de plantar 11.200 ha de floresta para suprir a demanda de lenha daquele ano.

Visto isso, o objetivo foi analisar a qualidade do carvão vegetal, para o consumo doméstico, comercializado em três municípios do Estado do Espírito Santo.

\section{MATERIAL E MÉTODOS}

A cada 30 dias foram coletadas quatro amostras de carvão vegetal disponíveis em quatro diferentes estabelecimentos dos seguintes municípios do Estado do Espírito Santo: Alegre; Jerônimo Monteiro e Cachoeiro de Itapemirim. Cada amostra de carvão vegetal possuía $2 \mathrm{Kg}$ descritos na embalagem. As amostras foram identificadas e encaminhadas ao Laboratório de Energia da Biomassa do Departamento de Engenharia Florestal do Centro de Ciências Agrárias da Universidade Federal do Espírito Santo (DEF/CCA/UFES), localizado no município de Jerônimo Monteiro/ES.

Foi realizada a análise química imediata do carvão conforme a Norma Brasileira Regulamentadora/ NBR 8112 (Associação Brasileira de Normas Técnicas/ ABNT, 1986).

A densidade relativa aparente foi determinada nas amostras utilizando o procedimento de imersão em água, descrito na NBR 11941 (ABNT, 2003).

$\mathrm{O}$ poder calorífico superior foi determinado por meio de um calorímetro conforme o procedimento descrito na NBR 8633 (ABNT, 1983) e a análise elementar (teor de carbono, hidrogênio, nitrogênio, enxofre e, por diferença, o teor de oxigênio), foram determinados no Laboratório de Energia da Biomassa do Departamento de Ciências Florestais da Universidade Federal de Lavras, localizado na município de Lavras/MG.

A análise estatística dos dados obtidos foi processada por meio do Sistema de Análises Estatísticas e Genéticas (SAEG), desenvolvido pelo Centro de Processamento de Dados da Universidade Federal de Viçosa. Foi utilizada a análise de variância (teste $\mathrm{F}$ ) a 5\% de significância e quando os dados foram significativos, foi empregado o teste de Tukey a 5\% de significância para a comparação de médias.

\section{RESULTADOS E DISCUSSÃO}

Para melhor entendimento dos resultados, cada estabelecimento e cada amostra coletada dos diferentes municípios foram identificadas de acordo com o Quadro 1.

Quadro 1. Identificação dos estabelecimentos e de cada amostra em função dos municípios.

\begin{tabular}{|c|c|c|}
\hline Município & Estabelecimento & Amostra \\
\hline \multirow{3}{*}{ Alegre (AI) } & $1 \mathrm{e} 4$ & $\mathrm{~A}$ \\
\cline { 2 - 3 } & 2 & $\mathrm{~B}$ \\
\cline { 2 - 3 } & 3 & $\mathrm{C}$ \\
\hline \multirow{3}{*}{$\begin{array}{c}\text { Jerônimo } \\
\text { Monteiro (JM) }\end{array}$} & 5 & $\mathrm{D}$ \\
\cline { 2 - 3 } & 7 & $\mathrm{E}$ \\
\hline \multirow{3}{*}{$\begin{array}{c}\text { Cachoeiro de } \\
\text { Itapemirim (CI) }\end{array}$} & $10,11 \mathrm{e} 12$ & $\mathrm{~A}$ \\
\cline { 2 - 3 } & 12 & $\mathrm{~F}$ \\
\hline
\end{tabular}

Como pode ser observado no Quadro 1, os estabelecimentos 1, 4 e 7, de municípios diferentes, comercializavam a amostra A. Os estabelecimentos 3, 10, 11 e 12 também de municípios distintos, comercializavam a mesma amostra C. Já o restante dos estabelecimentos comercializava amostras diferentes.

\section{Análise química imediata para cada município}

São apresentados na Tabela 1, os valores médios da densidade relativa aparente (D), umidade (U), teor de materiais voláteis (TMV), teor de carbono fixo (TCF), teor de cinzas (TCz) e poder calorífico superior (PCS) para as amostras coletadas em Alegre, Jerônimo Monteiro e Cachoeiro de Itapemirim. 
Tabela 1. Valores médios de densidade relativa aparente, umidade, análise química imediata e poder calorífico superior das amostras de carvão vegetal comercializado em Alegre, Jerônimo Monteiro e Cachoeiro de Itapemirim.

\begin{tabular}{|c|c|c|c|c|c|c|c|}
\hline Município & Amostra & $\mathrm{D}\left(\mathrm{g} \cdot \mathrm{cm}^{-3}\right)$ & $\mathrm{U}(\%)$ & $\operatorname{TMV}(\%)$ & $\mathrm{TCF}(\%)$ & $\mathrm{TCz}(\%)$ & PCS $\left(\right.$ cal.g $\left.{ }^{-1}\right)$ \\
\hline \multirow{8}{*}{$\mathrm{Al}$} & \multirow{2}{*}{ A } & 0,353 & 4,05 & $23,19 \mathrm{ab}$ & $75,90 a$ & 0,63 & 7270,00 \\
\hline & & $(0,08)^{* *}$ & $(01,24)$ & $(3,13)$ & $(3,22)$ & $(01,08)$ & $(223,62)$ \\
\hline & \multirow{2}{*}{ B } & 0,420 & 4,49 & $29,45 \mathrm{a}$ & $69,45 \mathrm{a}$ & 0,83 & 7292,00 \\
\hline & & $(0,12)$ & $(1,40)$ & $(6,78)$ & $(6,89)$ & $(0,95)$ & $(492,43)$ \\
\hline & \multirow{2}{*}{$\mathrm{C}$} & 0,425 & 4,60 & $21,92 \mathrm{~b}$ & $77,40 \mathrm{a}$ & 0,89 & 7475,00 \\
\hline & & $(0,04)$ & $(0,98)$ & $(3,84)$ & $(3,89)$ & $(1,02)$ & $(129,44)$ \\
\hline & \multirow{2}{*}{ Média } & 0,379 & $4,17 \mathrm{~B}$ & $23,64 \mathrm{~A}$ & $75,46 \mathrm{~B}$ & $0,68 \mathrm{~B}$ & $7432,00 \mathrm{~B}$ \\
\hline & & $(0,08)$ & $(1,21)$ & $(4,64)$ & $(4,68)$ & $(1,01)$ & $(281,67)$ \\
\hline \multirow{10}{*}{ JM } & \multirow{2}{*}{ D } & 0,382 & 4,25 & 22,94 & 75,78 & $1,23 \mathrm{~b}$ & 7340,50 \\
\hline & & $(0,02)^{* *}$ & $(1,05)$ & $(2,72)$ & $(4,33)$ & $(2,60)$ & $(261,53)$ \\
\hline & \multirow{2}{*}{$\mathrm{E}$} & 0,352 & 4,99 & 19,06 & 75,08 & $4,93 \mathrm{a}$ & 7387,00 \\
\hline & & $(0,15)$ & $(0,61)$ & $(2,86)$ & $(2,36)$ & $(0,52)$ & $(385,64)$ \\
\hline & \multirow{2}{*}{ A } & 0,372 & 4,09 & 22,65 & 75,60 & 1,60ab & 7436,50 \\
\hline & & $(0,08)$ & $(0,63)$ & $(4,01)$ & $(3,38)$ & $(0,69)$ & $(210,19)$ \\
\hline & \multirow{2}{*}{$\mathrm{F}$} & 0,459 & 4,40 & 23,34 & 75,02 & $1,65 \mathrm{ab}$ & 7456,50 \\
\hline & & $(0,99)$ & $(2,00)$ & $(4,65)$ & $(4,50)$ & $(0,14)$ & $(198,70)$ \\
\hline & \multirow{2}{*}{ Média } & 0,373 & $4,25 \mathrm{~B}$ & $21,27 \mathrm{~A}$ & $75,33 \mathrm{~B}$ & $1,65 \mathrm{~A}$ & $7436,50 \mathrm{~B}$ \\
\hline & & $(0,09)$ & $(0,93)$ & $(3,78)$ & $(3,25)$ & $(2,04)$ & $(254,96)$ \\
\hline \multirow{8}{*}{ CI } & \multirow{2}{*}{ G } & 0,359 & 5,10 & 18,36 & 80,67 & 0,98 & 7654,50 \\
\hline & & $(0,01)^{* *}$ & $(0,49)$ & $(3,30)$ & $(3,39)$ & $(0,14)$ & $(276,62)$ \\
\hline & \multirow{2}{*}{$\mathrm{H}$} & 0,396 & 4,06 & 23,36 & 74,50 & 2,13 & 7225,00 \\
\hline & & $* *$ & $* *$ & $* *$ & $* *$ & $* *$ & $* *$ \\
\hline & \multirow{2}{*}{$\mathrm{C}$} & 0,377 & 5,99 & 13,56 & 84,21 & 1,06 & 7882,00 \\
\hline & & $(0,02)$ & $(0,97)$ & $(5,38)$ & $(5,32)$ & $(0,94)$ & $(326,46)$ \\
\hline & \multirow{2}{*}{ Média } & 0,377 & $5,57 \mathrm{~A}$ & 15,65 B & $83,40 \mathrm{~A}$ & $1,03 \mathrm{AB}$ & $7827,50 \mathrm{~A}$ \\
\hline & & $(0,02)$ & $(1,01)$ & $(4,89)$ & $(4,90)$ & $(0,80)$ & $(322,87)$ \\
\hline
\end{tabular}

Os dados seguidos por uma mesma letra minúscula, em cada coluna, não diferem estatisticamente (Tukey, $p>0,05)$ As médias seguidas por uma mesma letra maiúscula, em cada coluna, não diferem estatisticamente (Tukey, p>0,05) ** Desvio padrão; *** Dados de somente uma amostra.

\section{Carvão vegetal comercializado no município de Alegre}

Os valores encontrados tanto para a densidade relativa aparente, com média de $0,379 \mathrm{~g} . \mathrm{cm}^{-3}$ quanto para a umidade de $4,17 \%$ são adequados para o consumo doméstico.

A densidade relativa aparente possui uma relação direta com a qualidade do carvão vegetal, ou seja, quanto maior a densidade do carvão vegetal, maior será o seu PCS, assim, maior a energia contida no material por unidade de massa, o que acarreta no maior rendimento do carvão vegetal (Brito e Barrichelo, 1977). Já a umidade, possui uma relação inversa com a qualidade do carvão vegetal, pois quanto maior a umidade, menor o rendimento gravimétrico do carvão. Isso ocorre porque a água absorve toda a energia que o carvão vegetal libera durante a queima, até que seja totalmente evaporada, então quanto maior a umidade, mais massa o carvão vegetal irá perder para evaporar toda a água contida na sua estrutura (Brahan, 2002). 
Verifica-se que tanto a média da densidade de 0,379 g. $\mathrm{cm}^{-3}$, quanto da umidade de $4,17 \%, \mathrm{TCz}$ de $0,68 \%$ e PCS de 7432 cal.g $^{-1}$ foram iguais estatisticamente para todas as amostras pelo teste F. A média das amostras do TCF de 75,46\%, apesar de apresentarem comportamento significativo no teste $\mathrm{F}$, foram iguais para $\mathrm{o}$ teste $\mathrm{t}$. Já a média do TMV foi maior para a amostra $\mathrm{B}$ e menor para a C, com média geral de $23,64 \%$.

Embora um baixo TMV seja desejado no carvão vegetal para o consumo doméstico, visando a menor quantidade de substâncias tóxicas liberada durante o preparo de alimentos, a ignição será dificultada pela baixa quantidade destes materiais voláteis (Brahan, 2002). Visto isso, as amostras A e $\mathrm{C}$ foram as que apresentaram os melhores valores para o consumo, além de estar de acordo com as especificações do Selo Premium.

Quando os parâmetros de qualidade do Selo Premium são comparados com cada amostra, verifica-se que apenas a amostra $B$ não seguiu os padrões exigidos pelo selo, devido ao alto TMV de baixo TCF.

\section{Carvão vegetal comercializado no município Jerônimo Monteiro}

No município de Jerônimo Monteiro, em que os dados estão contidos na Tabela 1 , somente a média do $\mathrm{TCz}$ de $1,65 \%$ foi significativo pelo teste $\mathrm{F}$, já os outros parâmetros apresentaram-se significativos.

A amostra $\mathrm{D}$ foi a que apresentou a menor média para $\mathrm{TCz}$ de 1,23\%, seguida das amostras A, F e $\mathrm{E}$, que apresentou a maior média pelo teste de Tukey.

Quando cada amostra é analisada particularmente com os parâmetros do Selo Premium, as amostras $\mathrm{A}, \mathrm{E}$ e $\mathrm{F}$ não seriam certificadas por causa do alto $\mathrm{TCz}$ apresentado.

\section{Carvão vegetal comercializado no município de Cachoeiro de Itapemirim}

Em Cachoeiro de Itapemirim, foi coletada somente uma amostra $\mathrm{H}$, pois ela não foi mais encontrada no estabelecimento 12 , que provavelmente parou de comercializá-la.

Como pode ser visualizado na Tabela 1 , as amostras $\mathrm{C}$ e $\mathrm{G}$ não diferiram estatisticamente entre si em suas características físicas e químicas. Entretanto, cabe ressaltar uma particularidade em relação a amostra $\mathrm{H}$, pois a única amostra coletada apresentou, em relação às outras amostras, alto TMV de 23,36\% e TCZ de 2,13\%, bem como baixo TCF de $74,5 \%$ e umidade de $4,06 \%$.

Ainda na Tabela 1, nenhuma das amostras comercializadas em Cachoeiro de Itapemirim receberia o Selo Premium de qualidade, pois as amostras C e $\mathrm{G}$ apresentaram umidade acima dos parâmetros estabelecidos pelo Selo, ou seja, 5,1 e $5,99 \%$ respectivamente, enquanto a amostra $\mathrm{H}$, apesar de possuir umidade satisfatória, apresentou baixo TCF e alto $\mathrm{TCz}$ em relação aos parâmetros da certificação.

Quando se compara o carvão vegetal disponível em cada município, verifica-se que a média das densidades de 0,379 g. $\mathrm{cm}^{-3}$ é igual estatisticamente pelo teste $\mathrm{F}$ a $5 \%$ de significância.

Estatisticamente, as amostras de carvão vegetal comercializado em Cachoeiro de Itapemirim, apresentaram em média um alto TCF, baixo TMV e o $\mathrm{TCz}$ foi equivalente às amostras dos outros dois municípios, contudo, apresentaram em média, amostras com elevada umidade. Já os municípios de Alegre e Jerônimo Monteiro apresentaram, em média, amostras de carvão vegetal com alto TMV e de TCz respectivamente. Visto isto e seguindo os parâmetros de qualidade do Selo Premium, nenhum dos municípios comercializa carvão vegetal com a qualidade adequada para o consumo doméstico.

Como podem ser visualizados na Tabela 2, os dados obtidos pela análise química imediata foram próximos dos encontrados por Ribeiro e Vale (2006) e Pimenta e Barcelos (2000). Entretanto, os dados diferem daqueles encontrados por Trugilho et al. (2001), Trugilho et al. (2005), Paula, Trugilho e Rezende (2009) e Arantes (2009). Em números absolutos, a única amostra que não está dentro dos limites do Selo Premium é a amostra B, devido ao baixo TCF.

\section{Análise elementar do carvão vegetal para cada município}

$\mathrm{Na}$ análise elementar são apresentados os teores de nitrogênio (N\%); carbono $(\mathrm{C} \%)$; relação carbono/nitrogênio $(\mathrm{C} / \mathrm{N})$; ; hidrogênio $(\mathrm{H})$; relação carbono/hidrogênio $(\mathrm{C} / \mathrm{H})$; enxofre $(\mathrm{S} \%)$ e oxigênio $(\mathrm{O} \%)$ das amostras de carvão vegetal comercializadas em Alegre, Jerônimo Monteiro e Cachoeiro de Itapemirim. 
Tabela 2. Valores médios de densidade relativa aparente, umidade, análise química imediata e poder calorífico superior.

\begin{tabular}{ccccccc}
\hline Autores & $\mathrm{D}\left(\mathrm{g} . \mathrm{cm}^{-3}\right)$ & $\mathrm{U}(\%)$ & $\mathrm{TMV}(\%)$ & $\mathrm{TCF}(\%)$ & $\mathrm{TCz}(\%)$ & $\mathrm{PCS}\left(\mathrm{cal.g}^{-1}\right)$ \\
\hline Pimenta e Barcelos (2000) & 0,46 & - & 21,03 & 75,06 & 3,91 & 8147,00 \\
\hline Trugilho et al. (2001) & $0,39-0,48$ & - & $18,05-23,50$ & $77,58-81,59$ & $0,25-0,51$ & - \\
\hline Selo Premium (2003) & - & $<5,00$ & $<23,50$ & $>75,00$ & $<1,5$ & - \\
\hline $\begin{array}{c}\text { Trugilho et al. (2005) } \\
\text { Ribeiro e Vale (2006) }\end{array}$ & $0,27-0,30$ & - & $30,04-32,08$ & $67,71-69,71$ & $0,29-0,37$ & - \\
\hline $\begin{array}{c}\text { Paula, Trugilho e Rezende } \\
\text { (2009) }\end{array}$ & - & 3,99 & 21,70 & 77,52 & 0,78 & 7524,53 \\
\hline $\begin{array}{c}\text { Arantes (2009) } \\
\text { Al }\end{array}$ & 0,36 & $3,54-4,41$ & $26,72-27,30$ & $72,54-73,03$ & $0,16-0,21$ & - \\
\hline $\begin{array}{c}\text { Rosa et al. } \\
\text { (2012) }\end{array}$ & 0,379 & 4,17 & 23,64 & 75,46 & 0,68 & 7432,00 \\
\hline
\end{tabular}

*Dados não apresentados pelos autores.

Carvão vegetal comercializado no município de Alegre

Como pode ser visualizado na Tabela 3 , a média dos teores de nitrogênio de $0,33 \%$; carbono de $80,58 \%$ e oxigênio de $15,54 \%$ não foram estatisticamente significativas, bem como as relações de $\mathrm{C} / \mathrm{N}$ de 254,45 e $\mathrm{C} / \mathrm{H}$ de 23,96. Todavia, as amostras $\mathrm{A}$ e $\mathrm{B}$ foram as que apresentaram estatisticamente, a maior e a menor média de hidrogênio (H\%) 3,71 e 3,25\% e enxofre (S\%) 0,19 e $0,03 \%$, respectivamente.

A caracterização elementar é importante, pois cada elemento químico possui uma característica particular. Por exemplo, o hidrogênio e o carbono cooperam positivamente para o poder calorífico, ao contrário do oxigênio. Já o enxofre e o nitrogênio contribuem negativamente para saúde humana e para o ambiente (Obemberger et al., 2005).

Pelos dados da análise química imediata, elementar e testes de média, foi correto afirmar que a amostra $\mathrm{A}$ demonstrou ser a mais adequada para o consumo, apresentando em média alto TCF e de $\mathrm{H}$, que contribui positivamente com o poder calorífico.

Carvão vegetal comercializado no município de Jerônimo Monteiro

A média dos teores de carbono de $80,76 \%$, hidrogênio de $3,02 \%$, enxofre de $0,03 \%$ e oxigênio de $15,89 \%$ não foram significativos estatisticamente pelo teste $\mathrm{F}$.

Contudo, as amostras D e E foram, estatisticamente, as que apresentaram o menor e maior teores de nitrogênio 0,19 e 0,39\%, respectivamente, pelo teste de Tukey (Tabela 2 ).

A média da relação $\mathrm{C} / \mathrm{N}$ de 280,60 foi menor para as amostras A, E e F, demonstrando que nestas amostras a liberação do dióxido de carbono é mais rápida, fato importante na ciclagem deste elemento pela natureza, conforme Shneider et al. (2005). Já a média da relação $\mathrm{C} / \mathrm{H}$ de 26,97 foi igual estatisticamente para todas as amostras e, quanto maior esta relação, maior a energia liberada pelo carvão vegetal, o que comprova o PCS ser também estatisticamente igual para todas as amostras.

De acordo com os dados da análise química imediata, elementar e testes de média, foi correto 
afirmar que a amostra $\mathrm{D}$ demonstrou ser a mais adequada para o consumo doméstico, pois, além de apresentar baixo TCZ, também apresentou a menor média de nitrogênio. Além disso, quando comparamos cada amostra em valores absolutos com os índices do Selo Premium, verifica-se que somente a amostra $\mathrm{D}$ segue tais padrões.

Tabela 3. Valores médios da análise elementar e das relações carbono/nitrogênio e carbono/hidrogênio das amostras de carvão vegetal comercializadas em Alegre, Jerônimo Monteiro e Cachoeiro de Itapemirim.

\begin{tabular}{|c|c|c|c|c|c|c|c|c|}
\hline Município & Amostra & $\mathrm{N}(\%)$ & $\mathrm{C}(\%)$ & $\mathrm{C} / \mathrm{N}$ & $\mathrm{H}(\%)$ & $\mathrm{C} / \mathrm{H}$ & S (\%) & $\mathrm{O}(\%)$ \\
\hline \multirow{5}{*}{$\mathrm{Al}$} & A & $\begin{array}{c}0,35 \\
(0,13)^{* *}\end{array}$ & $\begin{array}{l}80,74 \\
(3,72)\end{array}$ & $\begin{array}{l}259,53 \\
(91,32)\end{array}$ & $\begin{array}{l}3,25 \mathrm{a} \\
(0,32)\end{array}$ & $\begin{array}{l}25,10 \\
(3,38)\end{array}$ & $\begin{array}{l}0,06 \mathrm{~b} \\
(0,08)\end{array}$ & $\begin{array}{l}15,59 \\
(3,53)\end{array}$ \\
\hline & B & $\begin{array}{c}0,35 \\
(0,12)\end{array}$ & $\begin{array}{l}79,30 \\
(4,56)\end{array}$ & $\begin{array}{l}249,49 \\
(81,05)\end{array}$ & $\begin{array}{c}3,41 \mathrm{ab} \\
(0,30)\end{array}$ & $\begin{array}{l}23,46 \\
(3,28)\end{array}$ & $\begin{array}{l}0,03 \mathrm{~b} \\
(0,01)\end{array}$ & $\begin{array}{l}16,92 \\
(4,23)\end{array}$ \\
\hline & $\mathrm{C}$ & 0,47 & 78,86 & 180,18 & 3,71 & 21,71 & $0,19 \mathrm{a}$ & 16,78 \\
\hline & $C$ & $(0,07)$ & $(2,94)$ & $(42,58)$ & $(0,27) \mathrm{b}$ & $(2,32)$ & $(0,17)$ & $(2,66)$ \\
\hline & Média & $\begin{array}{l}0,33 \mathrm{~A} \\
(0,12)\end{array}$ & $\begin{array}{c}80,58 \mathrm{~B} \\
(3,66)\end{array}$ & $\begin{array}{c}254,45 \mathrm{~B} \\
(85,22)\end{array}$ & $\begin{array}{l}3,43 \mathrm{~A} \\
(0,34)\end{array}$ & $\begin{array}{c}23,96 \mathrm{~B} \\
(3,36)\end{array}$ & $\begin{array}{l}0,03 \mathrm{~A} \\
(0,11)\end{array}$ & $\begin{array}{l}15,54 \mathrm{~A} \\
(15,54)\end{array}$ \\
\hline \multirow{5}{*}{ JM } & D & $\begin{array}{c}0,19 \mathrm{~b} \\
(0,05)^{* *}\end{array}$ & $\begin{array}{l}80,99 \\
(1,64)\end{array}$ & $\begin{array}{c}451,17 \mathrm{a} \\
(167,77)\end{array}$ & $\begin{array}{c}3,08 \\
(0,27)\end{array}$ & $\begin{array}{l}26,65 \\
(2,74)\end{array}$ & $\begin{array}{c}0,02 \\
(0,03)\end{array}$ & $\begin{array}{l}15,89 \\
(1,51)\end{array}$ \\
\hline & $\mathrm{E}$ & $\begin{array}{l}0,39 \mathrm{a} \\
(0,21)\end{array}$ & $\begin{array}{l}81,62 \\
(4,52)\end{array}$ & $\begin{array}{c}212,56 \mathrm{ab} \\
(64,98)\end{array}$ & $\begin{array}{c}2,58 \\
(0,30)\end{array}$ & $\begin{array}{l}31,27 \\
(4,72)\end{array}$ & $\begin{array}{c}0,04 \\
(0,02)\end{array}$ & $\begin{array}{l}15,12 \\
(4,20)\end{array}$ \\
\hline & A & $\begin{array}{c}0,26 a b \\
(0,10)\end{array}$ & $\begin{array}{l}79,91 \\
(2,61)\end{array}$ & $\begin{array}{c}316,42 \mathrm{ab} \\
(183,31)\end{array}$ & $\begin{array}{c}3,2 \\
(0,33)\end{array}$ & $\begin{array}{l}25,02 \\
(3,48)\end{array}$ & $\begin{array}{c}0,04 \\
(0,02)\end{array}$ & $\begin{array}{l}16,57 \\
(2,40)\end{array}$ \\
\hline & $\mathrm{F}$ & $\begin{array}{c}0,30 \mathrm{ab} \\
(0,04)\end{array}$ & $\begin{array}{l}80,51 \\
(4,74)\end{array}$ & $\begin{array}{c}269,95 \mathrm{ab} \\
(22,38)\end{array}$ & $\begin{array}{c}3,2 \\
(0,67)\end{array}$ & $\begin{array}{l}25,86 \\
(6,87)\end{array}$ & $\begin{array}{c}0,02 \\
(0,04)\end{array}$ & $\begin{array}{l}15,97 \\
(4,12)\end{array}$ \\
\hline & Média & $\begin{array}{c}0,285 \mathrm{AB} \\
(0,17)\end{array}$ & $\begin{array}{c}80,76 \mathrm{AB} \\
(3,07)\end{array}$ & $\begin{array}{c}280,60 \mathrm{AB} \\
(176,53)\end{array}$ & $\begin{array}{l}3,02 \mathrm{~B} \\
(0,40)\end{array}$ & $\begin{array}{c}26,97 \mathrm{~B} \\
(4,51)\end{array}$ & $\begin{array}{l}0,03 \mathrm{~A} \\
(0,02)\end{array}$ & $\begin{array}{c}15,89 \mathrm{AB} \\
(2,79)\end{array}$ \\
\hline \multirow{4}{*}{ CI } & G & $\begin{array}{c}0,16 \mathrm{~b} \\
(0,02)^{* *}\end{array}$ & $\begin{array}{l}85,17 \\
(2,99)\end{array}$ & $\begin{array}{c}546,83 a \\
(70,93)\end{array}$ & $\begin{array}{c}2,59 \\
(0,45)\end{array}$ & $\begin{array}{l}32,92 \\
(6,63)\end{array}$ & $\begin{array}{c}0,02 \\
(0,01)\end{array}$ & $\begin{array}{l}12,00 \\
(2,68)\end{array}$ \\
\hline & $\mathrm{H}$ & $\begin{array}{l}0,40 \\
* * *\end{array}$ & $\begin{array}{c}77,36 \\
* * *\end{array}$ & $\begin{array}{c}193,40 \\
* * *\end{array}$ & $\begin{array}{l}3,11 \\
* * *\end{array}$ & $\begin{array}{c}24,91 \\
* * *\end{array}$ & $\begin{array}{l}0,04 \\
* * *\end{array}$ & $\begin{array}{c}19,09 \\
* * *\end{array}$ \\
\hline & C & $\begin{array}{c}0,24 \mathrm{ab} \\
(0,08)\end{array}$ & $\begin{array}{l}86,18 \\
(4,57) \\
\end{array}$ & $\begin{array}{c}352,86 \text { b } \\
(94,49)\end{array}$ & $\begin{array}{c}2,26 \\
(0,38) \\
\end{array}$ & $\begin{array}{l}38,67 \\
(6,25) \\
\end{array}$ & $\begin{array}{c}0,02 \\
(0,01)\end{array}$ & $\begin{array}{l}11,27 \\
(4,28)\end{array}$ \\
\hline & Média & $\begin{array}{c}0,225 \mathrm{~B} \\
(0,10)\end{array}$ & $\begin{array}{c}85,63 \mathrm{~A} \\
(4,35)\end{array}$ & $\begin{array}{l}372,51 \mathrm{~A} \\
(138,38)\end{array}$ & $\begin{array}{c}2,33 \mathrm{C} \\
(0,41)\end{array}$ & $\begin{array}{c}37,18 \mathrm{~A} \\
(6,53)\end{array}$ & $\begin{array}{l}0,02 \mathrm{~A} \\
(0,01)\end{array}$ & $\begin{array}{c}11,56 \mathrm{~B} \\
(4,01)\end{array}$ \\
\hline
\end{tabular}

Os dados seguidos por uma mesma letra minúscula, em cada coluna, não diferem estatisticamente (Tukey, p>0,05) As médias seguidas por uma mesma letra maiúscula, em cada coluna, não diferem estatisticamente (Tukey, p >0,05) ** Desvio padrão; *** Dados de somente uma amostra. 
Carvão vegetal comercializado no município Município de Cachoeiro de Itapemirim

Os teores de carbono, relação carbono/nitrogênio, relação carbono/hidrogênio, enxofre e oxigênio não foram significativos estatisticamente pelo teste F. Contudo, a amostra $\mathrm{C}$ foi a que apresentou a maior média do teor de nitrogênio, comparada à média da amostra $\mathrm{G}$.

A relação $\mathrm{C} / \mathrm{N}$ foi menor, estatisticamente, para a amostra $\mathrm{C}$, demonstrando que a liberação do dióxido de carbono é mais rápida nesta amostra, fato importante no ciclo deste elemento, pois quanto mais depressa ocorre esta liberação, mais rápido este componente volta para o seu ciclo natural e, mais rápido ocorre sua ciclagem, conforme Shneider et al. (2005).

No que tange a análise elementar (Tabela 3), a quantidade enxofre não foi estatisticamente significativa para o carvão vegetal dos três municípios. Entretanto, o município de Cachoeiro de Itapemirim foi o que apresentou o carvão vegetal com os menores teores de nitrogênio, hidrogênio, oxigênio e o maior valor de carbono, sendo esses teores inversos para as amostras do município de Alegre.

A relação $\mathrm{C} / \mathrm{N}$ foi maior para as amostras do município de Cachoeiro de Itapemirim, apresentando um carvão vegetal que demora a liberar nitrogênio para o ambiente, retardando a sua ciclagem natural, contudo, foi o município que também apresentou o carvão com a maior relação $\mathrm{C} / \mathrm{H}$, demonstrando comercializar um produto com alta capacidade calorífica.

Portanto é correto afirmar que, mesmo o município de Cachoeiro de Itapemirim comercializar amostras de carvão vegetal com alta umidade e valores elevados na relação $\mathrm{C} / \mathrm{N}$, foi o município que apresentou o carvão vegetal com os melhores resultados perante a análise química imediata, análise elementar e na relação $\mathrm{C} / \mathrm{H}$.

Contudo, vale ressaltar que, de acordo com os parâmetros de qualidade do Selo Premium, nenhum dos municípios comercializa carvão vegetal com a qualidade adequada para o consumo doméstico.

Os resultados foram próximos dos encontrados por Paula, Trugilho e Rezende (2009), que obtiveram valores para o $\mathrm{C}$ de $75 \%$; $\mathrm{H}$ de $3 \%$; $\mathrm{N}$ de $1 \% ; \mathrm{S}$ de 0,5\%; O de 17\%; $\mathrm{C} / \mathrm{N}$ de 75 e $\mathrm{C} / \mathrm{H}$ de 25 . Mas diferentes dos encontrados pela fundação Centro Tecnológico de Minas Gerais/ CETEC (2007), de $80,3 \%$ para o $\mathrm{C} ; 3,1 \%$ para o $\mathrm{H} ; 0,2 \%$ para o $\mathrm{N}$;
$0 \%$ para $\mathrm{S} ; 11,3 \%$ para $\mathrm{O} ; \mathrm{C} / \mathrm{N}$ de 401,5 e $\mathrm{C} / \mathrm{H}$ de 25,9 .

\section{CONCLUSÕES}

Os municípios de Alegre e Jerônimo Monteiro foram os que apresentaram o carvão vegetal de pior qualidade para o consumo doméstico.

As amostras de carvão vegetal disponíveis em Cachoeiro de Itapemirim apresentaram as melhores qualidades físicas e químicas quando comparado aos comercializados nos outros dois municípios, todavia, não atendendo às especificações do Selo Premium em razão da alta umidade.

O Selo Premium criado pelo Estado de São Paulo é um avanço nos direitos dos consumidores de carvão vegetal para o uso doméstico e poderia ser implementado em outros Estados da Federação.

\section{AGRADECIMENTO}

Ao Laboratório de Energia da Biomassa Florestal do Departamento de Ciências Florestais da Universidade Federal de Lavras.

\section{RESUMO}

O presente trabalho teve como objetivo analisar da qualidade do carvão vegetal, voltado para o consumo doméstico, comercializado nos seguintes municípios do Estado do Espírito Santo: Alegre; Jerônimo Monteiro e Cachoeiro de Itapemirim. Foram analisadas características do carvão vegetal, tais como a densidade relativa aparente, umidade, teor de materiais voláteis, teor de cinzas, teor de carbono fixo, poder calorífico superior, análise elementar e os resultados foram comparados com os parâmetros exigidos pelo Selo Premium do Estado de São Paulo. Verificou-se que, apenas uma amostra no município de Alegre e outra amostra no município de Jerônimo Monteiro não apresentaram qualidade satisfatória para o consumo doméstico. Já no município de Cachoeiro de Itapemirim, todas as amostras apresentaram melhores qualidades físicas e químicas, em média, quando comparado aos carvões comercializados nos outros dois municípios, todavia, não atendiam às especificações do Selo Premium em razão da alta umidade.

Palavras-chave: Carvão vegetal, qualidade, consumo doméstico

\section{REFERÊNCIAS}

Arantes, M. D. C. Variação das características da madeira e do carvão de um clone de Eucalyptus grandis W. Hill ex Maiden x Eucaylptus urophylla S. T. Blake. Tese (Doutorado em Ciência e 
Tecnologia da Madeira) - Universidade Federal de Lavras, 2009.

ASSOCIAÇÃO BRASILEIRA DE NORMAS TÉCNICAS - ABNT. NBR 8633, Rio de Janeiro, 1983.

ASSOCIAÇÃO BRASILEIRA DE NORMAS TÉCNICAS - ABNT. NBR 8112, Rio de Janeiro, 1986.

ASSOCIAÇÃO BRASILEIRA DE NORMAS TÉCNICAS - ABNT. NBR 11941, Rio de Janeiro, 2003.

Brahan, W. K. (2002), Combustibilidad de la madera: la experiência com espécies colombianas. 1. Bogotá: Fondo de Publicaciones, 50p.

Brito, J. O. and Barrichelo, L. E. G. (1977), Correlações entre características físicas e químicas da madeira e a produção de carvão vegetal: 1 densidade e teor de lignina da madeira de eucalipto. Instituto de Pesquisa e Estudos Florestais, 14, 9-20.

Coutinho, A. dos R.; Ferraz, E. S. B. (1988), Determinação da friabilidade do carvão vegetal em função do diâmetro das árvores e temperatura de carbonização. Instituto de Pesquisa e Estudos Florestais, Piracicaba, 38, 33-37.

Instituto Capixaba de Pesquisa, Assistência Técnica e Extensão Rural - INCAPER. Silvicultura, Vitória-Espírito Santo, [2003].

Fundação Centro Tecnológico de Minas Gerais CETEC. Uso da madeira para fins energéticos. Belo Horizonte, 1980.

Obernberger, I.; Brunner, T.; Barnthaler, G. (2005), Chemical properties of solid biofuelssignificance and impact. Biomass \& Bioenergy, 30, 973-982.
Paula, L. E. R.; Trugilho, P. F.; Rezende, R. N. (2009), Avaliação de uma amostra de carvão vegetal de Eucalyptus para uso doméstico comercializado em Lavras/MG. Remade, 67, 1518.

Pennise, D. M.; Smith, K. R.; Kithinji, J. P.; Rezende, M. E. Raad, T. J.; Zhang, J.; Fan, C. (2001), Emissions of greenhouse gases and other airborne pollutants form charcoal making in Kenya and Brazil. Journal of Geophysical Research, 106, 2414-2415.

Pimenta, A. S and Barcellos, D. C. (2000), Curso de atualização e carvão vegetal. Centro de Produções técnicas - CPT, 76p.

Ribeiro, P. G. and Vale, A. T. (2006), Qualidade do carvão vegetal de resíduos de serraria para o uso doméstico. In: Reunião Anual da Sociedade Brasileira para o Progresso da Ciência, Florianópolis, 2006. Anais. Belém: Universidade Federal do Paraná.

São Paulo (Estado). (2003), Secretaria de Agricultura e Abastecimento de São Paulo. Resolução $n^{\circ} 10$ SAA, de 11 de julho de 2003. São Paulo.

Schneider, P. R.; Finger, C. A. G.; Sobrinho, V. G.; Schneider, P. S. P. (2005), Determinação indireta do estoque de biomassa e carbono em povoamentos de Acácia-negra (Acacia mearnsii De Wild.). Ciência Florestal, 15, 391-402.

Trugilho, P. F.; Lima, J. T.; Mori, F. A.; Lino, A. L. (2001), Avaliação de clones de Eucalyptus para produção de carvão vegetal. Cerne, 7, 104-114.

Trugilho, P. F.; Silva, J. R. M.; Mori, F. A.; Lima, J. T.; Mendes, L. M.; Mendes, L. F. B. (2005), Rendimentos e características do carvão vegetal em função da posição radial de amostragem em clones de Eucalyptus. Cerne, 11, 178-186. 